\title{
Epigenetics at the epicenter of a revolution in ARTs
}

\author{
David F. Albertini ${ }^{1}$ \\ Published online: 26 June 2018 \\ (C) Springer Science+Business Media, LLC, part of Springer Nature 2018
}

It's not what you look at that matters, it's what you see. Henry David Thoreau

Every once in a while, papers come through the JARG escalator in a distinct clustering of topics. So it was several months ago that the subject of epigenetics sprung forth, resulting in the current issue - the contents of which we will delve into later. Discriminating between fad, fancy, and frenzy became a matter of principle, especially within the context of ARTs and genetics. The charge then became one of updating the knowledge base for our readership so that this rapidly expanding field of biology could take its rightful place in the annals of reproductive medicine.

With this backdrop, we draw attention to the article by Meloni and Testa, recognizing without equivocation that epigenetics has spawned a revolution in thinking for those enthralled with the interface between genetics, development, and evolution [1]. And we practitioners of reproductive medicine, while avoiding the latter component of this trio of disciplines, should take solace in their message of ambiguity when it comes to trying to define what epigenetics is at a molecular level, and how if ever solved, this would pertain to the practice of ARTs and offspring health - a notable but consistent concern for more than a decade now. So what is the vernacular of the molecular in the world of epigenetics? And how will this new knowledge influence the current and future practices of infertility treatment?

To get the conversation started, speaking the same language becomes a priority, and thankfully, this task has been undertaken in exemplary style by Zhu et al. [2]. Well beyond the gender-specific methylation marks of yesteryear through the reproductive life cycle of mammals, any number of RNA

David F. Albertini

eicjarg@gmail.com

1 Center for Human Reproduction, New York, NY, USA and DNA modifications that are enriching the alphabet soup of epigenetics and uncovering the impact of higher order alterations in chromatin have emerged. One such example at a mechanistic level is demonstrated in the paper by Krieg and colleagues from the University of Kansas Medical Center (Histone demethylase KDM4A and KDM4B expression in granulosa cells from women undergoing in vitro fertilization. https://doi.org/10.1007/s10815-018-1151). Here, the language usage seems not to pertain the oocyte genome itself but rather in the genomes of somatic granulosa cells hinting at the importance of maintaining methylation marks in IVF cycles that resulted in a pregnancy. Much more work is needed to begin to clarify the molecular signatures characteristic of gametes, embryos, and their somatic counterparts during the course of normal and impaired reproduction. And consonant with this translation (sic conversation) of molecular terminology is a parallel need to solve the riddle of inheritance of disease states through mechanisms not involving our outdated notion of how genes work [3].

The problem of inheritance through epigenetic alterations of genomes has attracted much attention from within and without the community of scientists investigating how changes in our environment - particularly with respect to endocrine disruptors - serve as a platform for transmission of "altered states" to offspring of exposed organisms [4]. As illustrated by the career efforts of Michael Skinner, the vicious cycle of environment to epimutation to mutation sets the stage for increasing the risks of disease or developmental disturbances when environmental exposures take their toll on germ cells or embryos at early stages of development [5]. As the role of environmental factors continues to loom large in the conversation regarding epigenetics and patterns of inheritance of the non-genetic variety [6], what is happening in the realm of human ARTs remains a bit of a puzzle. At least until now.

Many of the papers comprising this issue take our readership on a short ride through the world of epigenetics and 
human ARTs. From the review by Cortesis and colleagues (Comprehensive meta-analysis reveals association between multiple imprinting disorders and conception by assisted reproductive technology), to a consideration of the fetal origins of adult disease by Lubinsky (An epigenetic association of malformations, adverse reproductive outcomes, and fetal origins hypothesis related effects), to old and new insights into the incidence of imprinting disorders in IVF children (Detection of a case of Angelman syndrome caused by an imprinting error in 949 pregnancies analyzed for AS following $I V F$ ), we hope our readers will gain a deeper appreciation for the role of epigenetics in reproductive medicine.

As with all new areas of exploration begging for the skills of the critical observer to sort fact from fallacy, in the end, it will be what we see that matters the most!

\section{References}

1. Meloni M, Testa G. Scrutinizing the epigenetics revolution. Biosocieties. 2014;9(4):431-56.

2. Zhu Q, Stoger R, Alberio R. A lexicon of DNA modifications: their roles in embryo development and the germline. Front Cell Dev Biol. 2018;6:24.

3. Lane N. Heredity beyond the gene. Nature. 2018;557(7706):489-90.

4. Nilsson EE, Skinner MK. Environmentally induced epigenetic transgenerational inheritance of reproductive disease. Biol Reprod. 2015;93(6):145.

5. Skinner MK, Guerrero-Bosagna C, Haque MM. Environmentally induced epigenetic transgenerational inheritance of sperm epimutations promote genetic mutations. Epigenetics. 2015;10(8): 762-71.

6. Rothstein MA, Harrell HL, Marchant GE. Transgenerational epigenetics and environmental justice. Environ Epigenet. 2017;3(3): $\operatorname{dvx} 011$. 